\title{
Abordagem multidisciplinar no tratamento de atresia maxilar em odontopediatria:
}

\section{relato de caso}

\author{
Multidisciplinary approach for the treatment of maxillary atresia in pediatric dentistry: case report \\ Enfoque multidisciplinar en el tratamiento de la atresia maxilar en odontología pediátrica: reporte
}

de caso

Recebido: 19/12/2021 | Revisado: 27/12/2021 | Aceito: 31/12/2021 | Publicado: 08/01/2022

\author{
Luisiane de Avila Silva \\ ORCID: https://orcid.org/0000-0003-2991-8918 \\ Faculdade Ciências Médicas da Paraíba, Brasil \\ E-mail: luisianeavila@gmail.com \\ Priscila Hernández de Campos \\ ORCID: https://orcid.org/0000-0003-0655-3485 \\ Universidade Cruzeiro do Sul, Brasil \\ E-mail: hcampospriscila@yahoo.com.br \\ Alba Valeska Alves de Oliveira \\ ORCID: https://orcid.org/0000-0003-0396-5505 \\ Faculdade COESP, Brasil \\ E-mail: valeska-oliveira@hotmail.com \\ Michele Baffi Diniz \\ ORCID: https://orcid.org/0000-0002-0693-2162 \\ Universidade Cruzeiro do Sul, Brasil \\ E-mail: mibdiniz@hotmail.com
}

\begin{abstract}
Resumo
O objetivo deste caso clínico foi relatar a importância da ortodontia interceptativa associada ao acompanhamento otorrinolaringológico e fonoaudiológico em uma criança com diagnóstico de atresia dos maxilares. Paciente leucoderma, 5 anos de idade, sexo feminino, procurou atendimento odontológico com queixa de apinhamento dentário. A avaliação clínica e a documentação ortodôntica evidenciaram relação molar Classe I, relação canina Classe III, perfil convexo, retrusão do incisivo inferior, profundidade facial com retrusão mandibular, profundidade da maxila com retrusão maxilar e comprimento craniano anterior pequeno. A criança foi avaliada por um médico otorrinolaringologista que diagnosticou hipertrofia de adenoides, com saída de ar limítrofe, sem indicação de adenoidectomia. O tratamento proposto foi a disjunção da maxila com aparelho palatino de Haas associada à expansão lenta da mandíbula com um aparelho expansor removível. No mesmo período, a criança iniciou tratamento fonoaudiológico para restabelecimento das funções orofaciais, pois apresentava baixa tonicidade das bochechas e do lábio inferior com eversão discreta, lábio superior encurtado e hipertrofia do mento. Quanto à evolução clínica da criança, notou-se aumento do tônus orofacial de lábios e bochechas e diminuição do tônus do mentual, melhor padrão mastigatório alternado com fechamento labial mais frequente e melhor produção dos bilabiais durante a fala espontânea. Pôde-se concluir que a expansão rápida da maxila associada à expansão lenta da mandíbula foram efetivas no tratamento da criança, proporcionando bons resultados na deficiência da dimensão transversal. Além disso, a abordagem multiprofissional com Odontopediatria, Otorrinolaringologia e Fonoaudiologia contribuiu para o sucesso do processo terapêutico da criança.
\end{abstract}

Palavras-chave: Maxila; Técnica de Expansão Palatina; Ortodontia interceptora; Fonoaudiologia; Criança.

\begin{abstract}
The aim of this clinical case was to report the importance of interceptive orthodontics associated with otorhinolaryngological and speech therapy follow-up in a child diagnosed with maxillary atresia. A 5-year-old white female patient sought dental care complaining of tooth crowding. Clinical evaluation and orthodontic documentation showed Class I molar relationship, Class III canine relationship, convex profile, mandibular incisor retrusion, facial depth with mandibular retrusion, maxillary depth with maxillary retrusion, and small anterior cranial length. The child was evaluated by an otolaryngologist who diagnosed adenoid hypertrophy with borderline airflow, and without indication for adenoidectomy. The proposed treatment was maxillary disjunction with Haas palatine appliance associated with slow expansion of the mandible with a removable expander appliance. In the same period, the child started speech therapy to restore orofacial functions, as she presented low tone of the cheeks and lower lip with mild eversion, shortened upper lip and chin hypertrophy. As for the clinical evolution of the child, there was an increase in orofacial tonus of the lips and cheeks and a decrease in the tonus of the mentalis, a better chewing pattern alternated with more frequent lip closure and better bilabial production during spontaneous speech. It can be concluded that the
\end{abstract}


rapid expansion of the maxilla associated with the slow expansion of the mandible were effective in the treatment of the child, providing good results in the deficiency of the transversal dimension. Moreover, the multidisciplinary approach with Pediatric Dentistry, Otorhinolaryngology and Speech Therapy contributed to the success of the child's therapeutic process.

Keywords: Maxilla; Palatal Expansion Technique; Orthodontics, interceptive; Speech, language and hearing sciences; Child.

\section{Resumen}

El objetivo de este caso clínico fue informar sobre la importancia de la ortodoncia interceptiva asociada al seguimiento otorrinolaringológico y logopédico en un niño diagnosticado de atresia maxilar. Una paciente blanca de 5 años de edad buscó atención dental quejándose de apiñamiento de dientes. La evaluación clínica y la documentación de ortodoncia mostraron relación molar Clase I, relación canina Clase III, perfil convexo, retrusión del incisivo mandibular, profundidad facial con retrusión mandibular, profundidad maxilar con retrusión maxilar y longitud craneal anterior pequeña. La niña fue evaluada por un otorrinolaringólogo que le diagnosticó hipertrofia adenoidea, con flujo aéreo limítrofe, sin indicación de adenoidectomía. El tratamiento propuesto fue la disyunción maxilar con aparato palatino de Haas asociado con la expansión lenta de la mandíbula con un aparato expansor extraíble. En el mismo período, la niña inició logopedia para restaurar las funciones orofaciales, ya que presentaba tono bajo de mejillas y labio inferior con leve eversión, labio superior acortado e hipertrofia de mentón. En cuanto a la evolución clínica de la niña, se notó un aumento del tono orofacial de los labios y mejillas y una disminución del tono del mentón, un mejor patrón de masticación alternado con un cierre de labios más frecuente y una mejor producción bilabial durante habla espontánea. Se pudo concluir que la rápida expansión del maxilar asociada a la lenta expansión de la mandíbula fueron efectivas en el tratamiento del niño, brindando buenos resultados en la deficiencia de la dimensión transversal. Además, el abordaje multidisciplinario con Odontopediatría, Otorrinolaringología y Logopedia contribuyó al éxito del proceso terapéutico de la niña.

Palabras clave: Maxilar; Técnica de Expansión Palatina; Ortodoncia interceptiva; Fonoaudiología; Niño.

\section{Introdução}

A atresia maxilar (AM) é uma alteração do crescimento facial que ocasiona estreitamento da arcada superior no sentido horizontal. Esta assume uma forma triangular, a qual acarreta modificações funcionais e oclusais que podem ser esqueléticas ou dentárias, uni ou bilaterais e, raramente, tem resolução espontânea (Scariot et al., 2020). Essa deformidade apresenta origem multifatorial, podendo ter causas genéticas ou ambientais, uma vez que muitos arcos dentários atrésicos são fruto de hábitos bucais deletérios e respiração bucal (Andrade et al., 2020).

O tratamento não cirúrgico da atresia maxilar consiste na expansão do arco maxilar, que pode ser lenta ou rápida. A expansão lenta é comumente realizada para gerar menos resistência do tecido em torno das estruturas maxilares e, portanto, buscando melhorar a formação óssea na sutura intermaxilar, reduzindo os efeitos relacionados à força da expansão rápida. Acredita-se que a abertura da sutura óssea seja maior quanto menor for a idade do paciente (Almeida et al., 2017; Ìsil et al., 2017; Ferreira et al., 2021)

Assim, a expansão rápida da maxila (ERM) é um procedimento ortopédico mecânico eficiente e permanente utilizado para o tratamento das alterações transversais da maxila. Nessa abordagem terapêutica ocorre a separação da sutura palatina mediana (efeito esquelético) em um curto espaço de tempo. A abertura de um diastema entre os incisivos centrais superiores representa o efeito ortodôntico mais característico da ERM (Lima Filho, 2009).

Os aparelhos comumente usados na ERM são o aparelho disjuntor palatino de Haas, o aparelho de Hyrax e o aparelho McNamara. O aparelho disjuntor palatino de Haas tenta distribuir as forças geradas na ativação entre os dentes e a porção palatina da maxila com o acréscimo de acrílico no palato. Entretanto, com a gengiva marginal aliviada, apresenta mais benefícios para o tratamento das más-oclusões de Classe III, casos de AM, e auxilia que respiradores bucais se transformem em respiradores nasais (Caldas et al., 2019).

Diante do exposto, o objetivo do presente caso clínico foi relatar a importância da ortodontia interceptativa associada ao acompanhamento otorrinolaringológico e fonoaudiológico em uma criança de 5 anos de idade, apresentando o tratamento de atresia maxilar com o uso do aparelho disjuntor palatino de Haas e aparelho expansor removível inferior. 


\section{Metodologia}

Desenvolveu-se um estudo de caso clínico de cunho analítico descritivo (Pereira et al., 2018). Nesse trabalho a identidade do paciente foi preservada e foi assinado o Termo de Consentimento Livre e Esclarecido -TCLE pelos responsáveis. Após, iniciou-se a avaliação por exame clínico, anamnese, modelos de estudo e exames de imagem (radiografias periapicais e panorâmica). Associada a este levantamento de informações do caso foi realizada uma busca literária nos bancos de dados como Pubmed, Scielo e CAPES, realizando uma avaliação dos artigos que versavam sobre a temática abordada nesse estudo e de acordo com as palavras-chaves utilizadas no trabalho.

\section{Resultados e Discussão}

Paciente do sexo feminino, leucoderma, 5 anos e 6 meses de idade, procurou atendimento odontológico na Clínica de Odontopediatria na Faculdade COESP, em João Pessoa-PB, acompanhada de seu responsável, em maio de 2019. A queixa principal foi o apinhamento dentário e o histórico familiar de tratamento ortopédico. Na anamnese, o responsável relatou o hábito de respiração bucal, uso de mamadeira a partir dos 6 meses de idade três vezes ao dia até os 3 anos de idade. No entanto, negou uso de chupeta ou sucção digital.

Cabe ressaltar que, um mês antes da primeira consulta de avaliação odontológica, a paciente foi avaliada por um médico otorrinolaringologista, pois apresentava crises alérgicas recorrentes e sem causa definida. O profissional diagnosticou hipertrofia de adenoides, com saída de ar limítrofe, sem indicação de adenoidectomia ou outro tipo de tratamento.

Foi solicitada a documentação ortodôntica completa com análise cefalométrica computadorizada de Ricketts para diagnóstico e elaboração do plano de tratamento. Ao exame clínico intraoral, observou-se dentição decídua completa. A paciente apresentava relação molar Classe I, relação canina Classe III, perfil convexo, bom posicionamento da protrusão do incisivo superior, retrusão do incisivo inferior, lábio inferior protuso, profundidade facial com retrusão mandibular, profundidade da maxila com retrusão maxilar e o comprimento craniano anterior pequeno (Figura 1). 
Figura 1. Fotografias iniciais do perfil da paciente. (A) Perfil do lado direito. (B) Frente sem selamento labial. (C) Frente sorrindo. (D) Perfil do lado esquerdo.
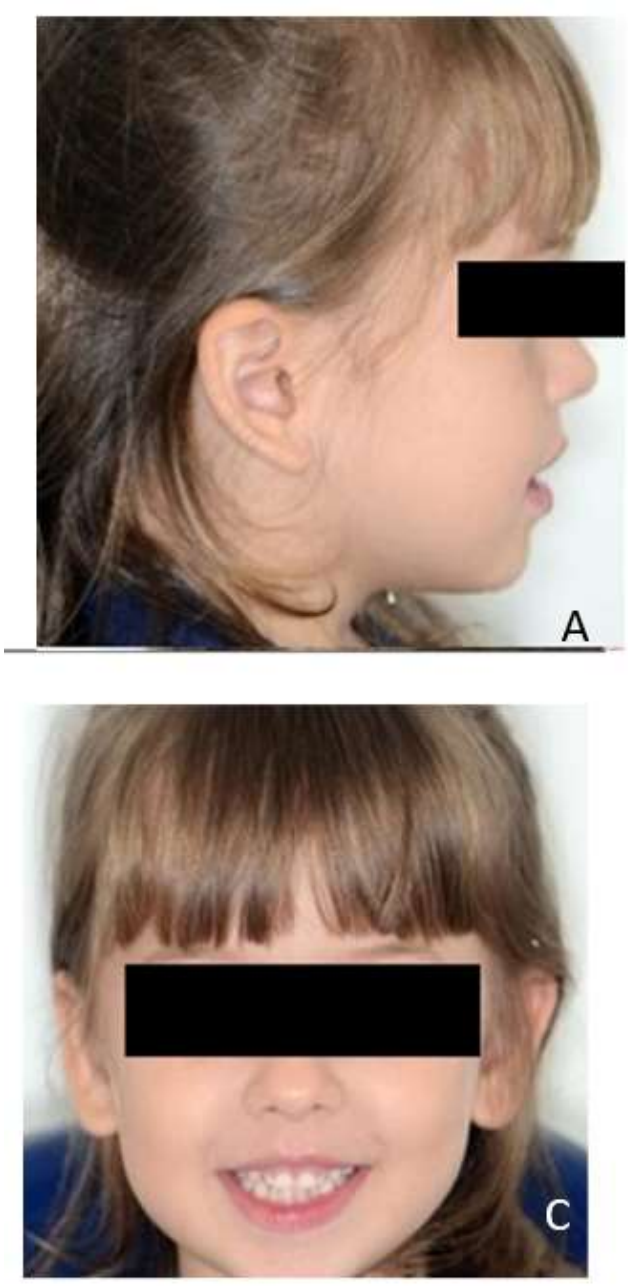
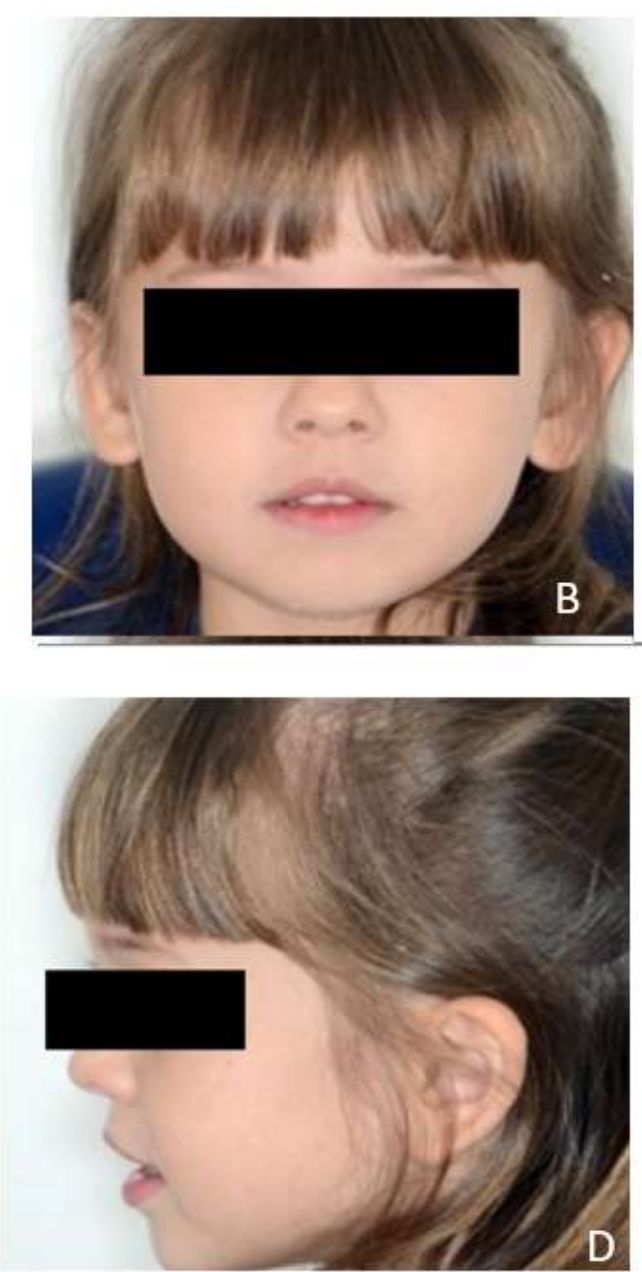

Fonte: Autores.

Notar o perfil convexo, o lábio inferior protuso, a profundidade facial com retrusão mandibular, a profundidade da maxila com retrusão maxilar e o comprimento craniano anterior pequeno.

Para o presente caso, o tratamento proposto foi a disjunção da maxila com aparelho disjuntor palatino de Haas associada à expansão lenta da mandíbula com um aparelho expansor removível. Os pais autorizaram o plano de tratamento e documentação do caso, assinando um Termo de Consentimento Livre e Esclarecido. O tratamento teve início em agosto de 2019, com a adaptação das bandas ortodônticas (Morelli, Sorocaba, SP, Brasil) nos dentes 55 e 65, seguido de moldagem de transferência e envio do modelo em gesso especial tipo IV (Polidental, Cotia, SP, Brasil) ao laboratório.

O aparelho disjuntor palatino de Haas foi instalado em setembro de 2019. As bandas ortodônticas foram cimentadas com cimento de ionômero de vidro modificado por resina Riva Light Cure (SDI, Victoria, Austrália) e a parte da estrutura metálica apoiada sobre os dentes foi fixada com resina composta Charisma Classic cor A2 (Kulzer, Wehrheim, Alemanha), com condicionamento ácido prévio em esmalte com ácido fosfórico a 37\% (Maquira, Maringá, PR, Brasil) e sistema adesivo Adper Single Bond (3M/ESPE, 3M do Brasil, Sumaré-SP, Brasil), seguido da realização da radiografia oclusal de acompanhamento do caso (Figuras 2 e 3). A criança e os responsáveis foram orientados quanto à higiene bucal e sobre ativação do disjuntor: a partir do segundo dia, ativação diária de $2 / 4$ de volta, sendo 1/4 de volta no período matutino e 1/4 de volta no período noturno. As ativações foram realizadas durante 14 dias. 
Figura 2. Instalação do aparelho disjuntor palatino de Haas. (A) Oclusão do lado direito. (B) Oclusão frontal. (C) Oclusão do lado esquerdo.
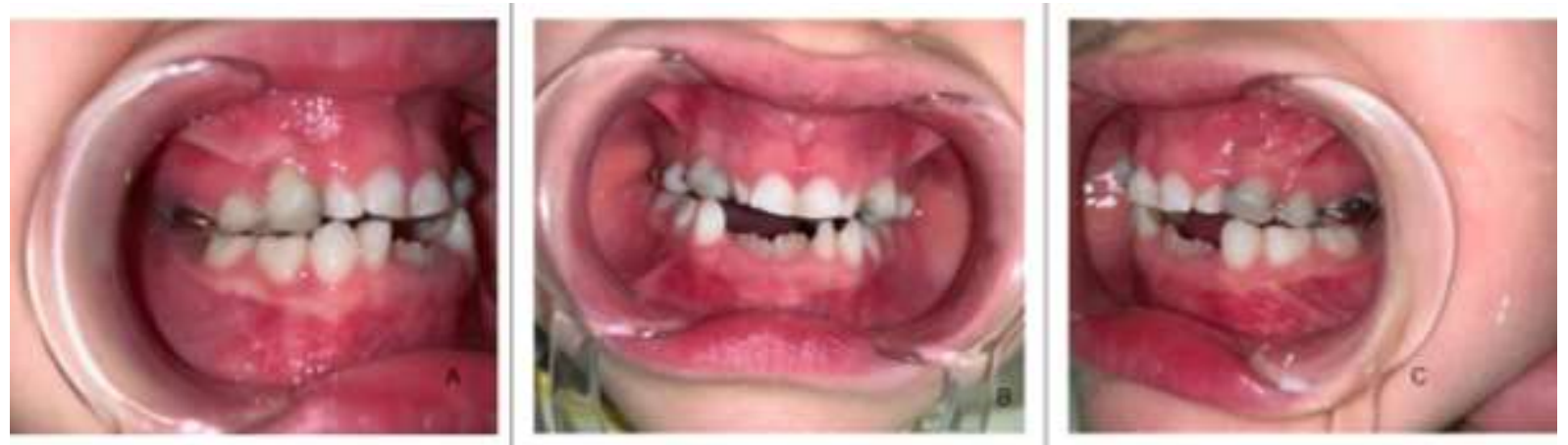

Fonte: Autores.

Observar o aparelho disjuntor palatino de Hass instalado.

Figura 3. (A) Radiografia oclusal inicial imediatamente após a instalação do aparelho disjuntor palatino de Haas. (B) Fotografia intrabucal mostrando a adaptação do aparelho.
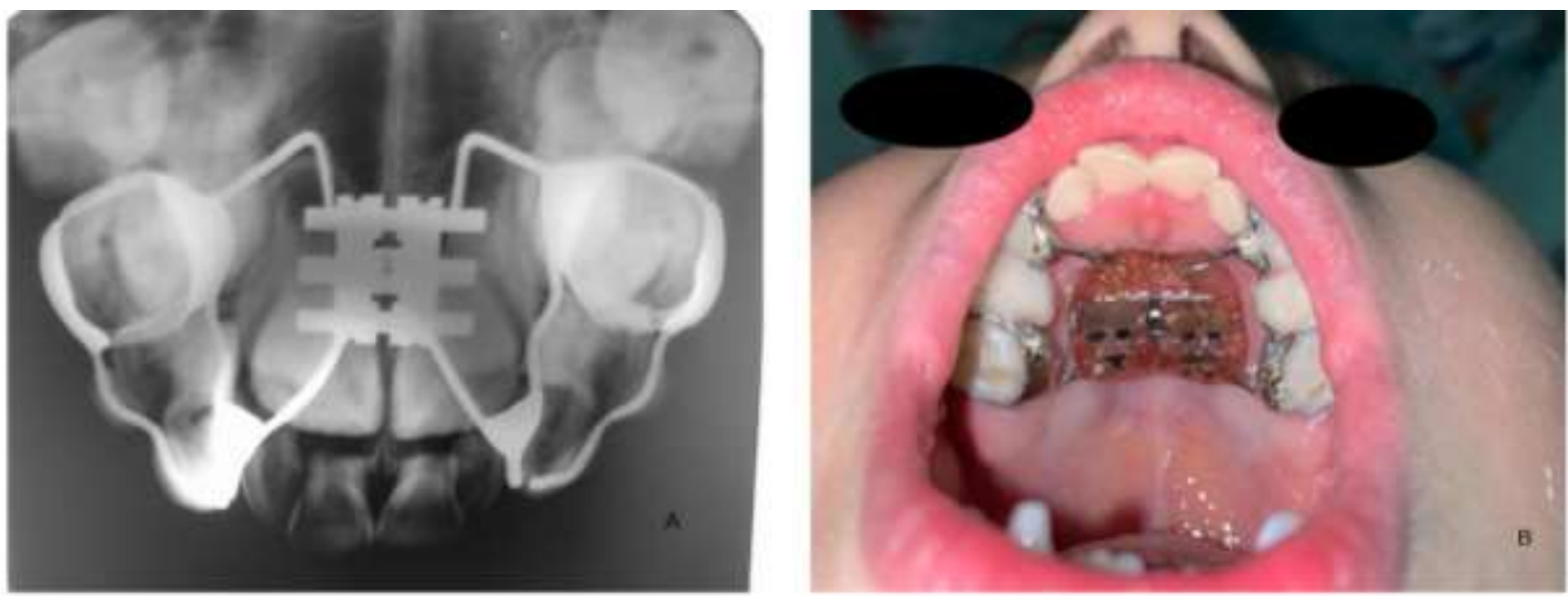

Fonte: Autores.

Notar a sutura palatina antes da disjunção e a personalização do aparelho disjuntor palatino de Hass para melhor adesão ao tratamento.

A instalação do aparelho expansor removível inferior foi realizada em novembro de 2019, a fim de proporcionar a expansão lenta da mandíbula para acompanhar a expansão rápida na maxila. A ativação era realizada a cada 15 dias (Figura 4). 
Figura 4. (A - B) Instalação do aparelho expansor removível inferior com recobrimento oclusal e arco de Hawley.
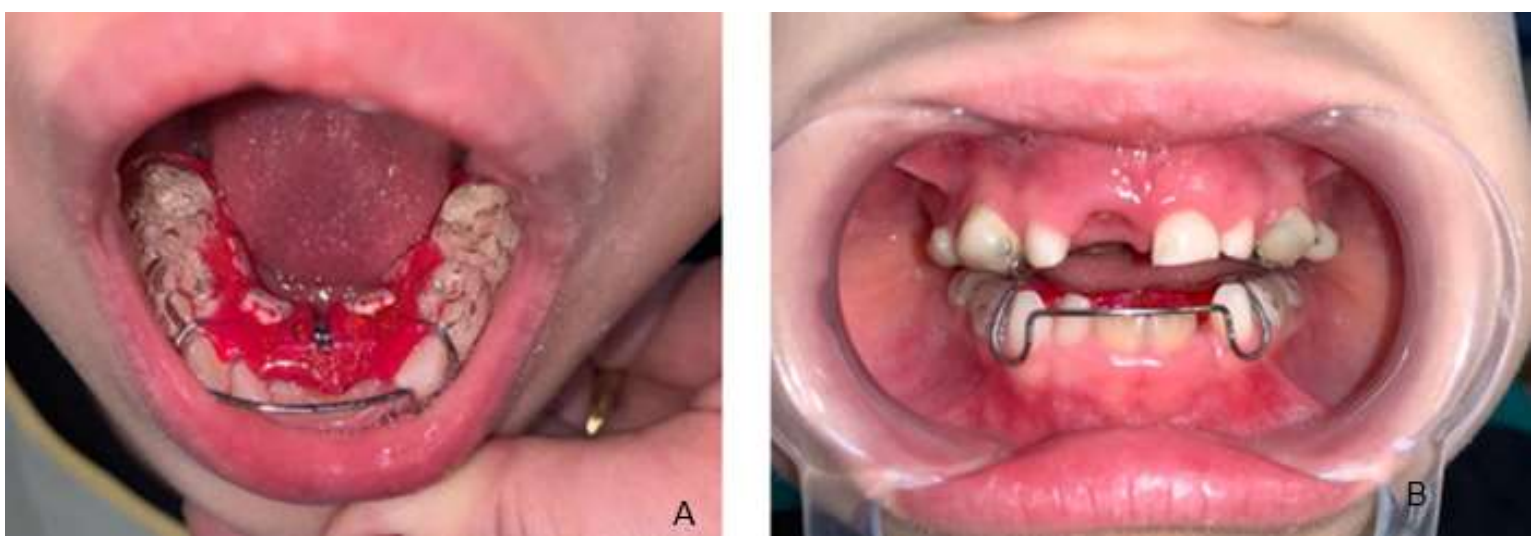

Fonte: Autores.

Observa-se o aparelho expansor removível inferior perfeitamente instalado no arco mandibular.

No mesmo período, a criança iniciou tratamento fonoaudiológico, pois apresentava baixa tonicidade das bochechas e do lábio inferior com eversão discreta, lábio superior encurtado e hipertrofia do mento. O planejamento terapêutico fonoaudiológico teve como objetivos conscientizar a paciente sobre a importância da respiração nasal; restabelecer as funções orofaciais (respiração nasal e sua automatização; mastigação); melhorar a postura labial, o tônus orofacial e orientar a família. Nas consultas foram realizados exercícios mioterápicos (isotônicos, isométricos e isocinéticos), massagens e alongamentos orofaciais, como também treino mastigatório (com o uso de alimentos) e da respiração nasal (com conscientização e propriocepção, cheiros, espelho de Glatzel e outros recursos da fonoaudiologia), além da aplicação de bandagem elástica.

Quanto à evolução clínica, a paciente apresentou aumento do tônus orofacial de lábios e bochechas e diminuição do tônus do mentual, o que contribuiu para uma melhor postura dos órgãos fonoarticulatórios (OFA) e vedamento labial em repouso. Quanto à mastigação, após o treinamento e orientações, observou-se melhor padrão mastigatório alternado com fechamento labial mais frequente. Na fonética, apresentou melhor produção dos bilabiais durante a fala espontânea (Figura 5). 
Figura 5. Fotografias finais do perfil da paciente. (A) Perfil do lado direito. (B) Perfil frontal sem selamento labial. (C) Perfil frontal sorrindo. (D) Perfil lateral.
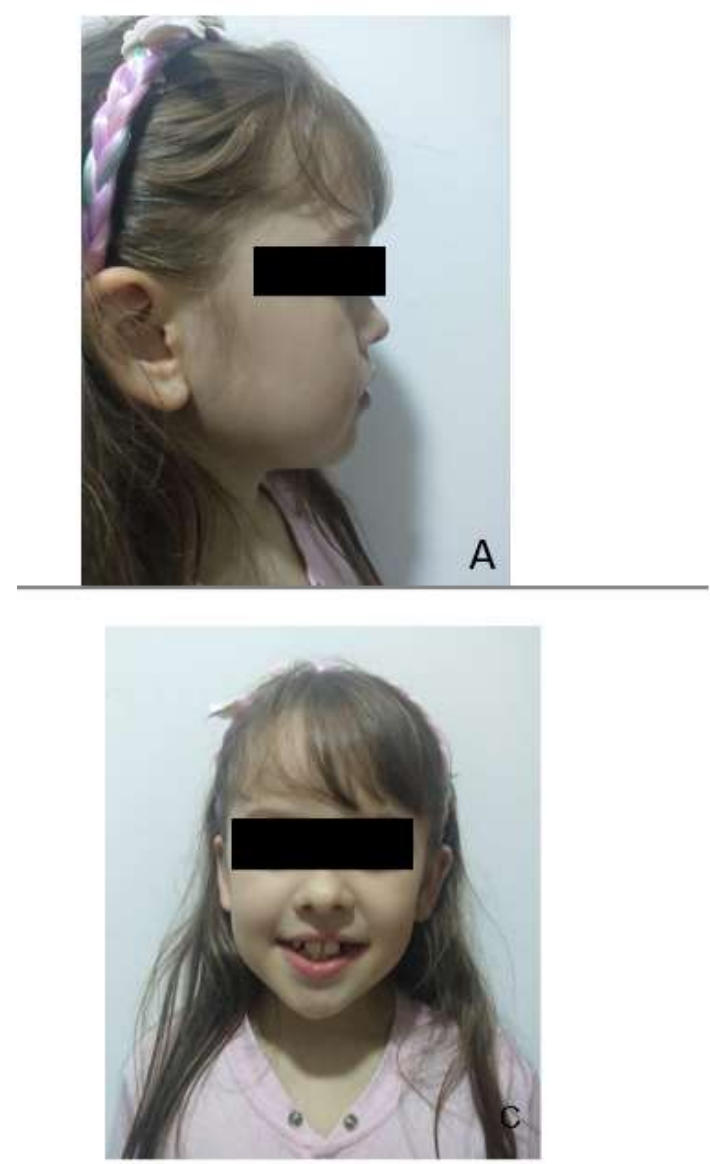
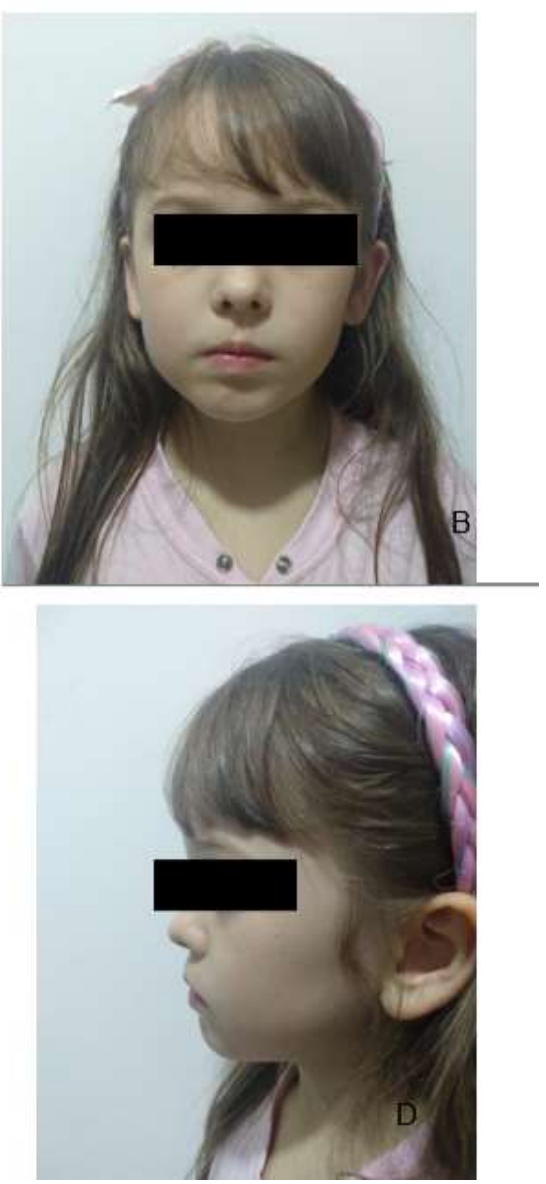

Fonte: Autores.

\section{Considerações Finais}

Pôde-se concluir que a ERM associada à expansão lenta da mandíbula foram efetivas no tratamento da criança, proporcionando bons resultados para a correção da deficiência da dimensão transversal. Além disso, a abordagem multiprofissional com o acompanhamento otorrinolaringológico e fonoaudiológico teve importante colaboração no processo terapêutico.

Salienta-se a importância do entendimento da origem multifatorial da atresia transversal da maxila e que a abordagem ideal deve ser avaliada pelo cirurgião-dentista de acordo com cada caso, com a escolha do disjuntor justificada de acordo com as características de cada paciente. Os efeitos da ERM estão relacionados com o tipo facial, respiração, ortopedia e fonação. Logo, a abordagem multidisciplinar permite o manejo conjunto de todos os fatores envolvidos. Nesse sentido, são necessários mais estudos e publicações com o intuito de aprofundar os conhecimentos sobre essa temática.

\section{Referências}

de Almeida, T. E., Saavedra, J., Pavlovsky, M., Scrocco, J. A., dos Santos, M. G., \& Monteiro, C. G. (2017). Expansão rápida da maxila não cirúrgica e cirúrgica: revisão de literatura. Revista de Odontologia da Universidade Cidade de São Paulo, 24(1), 67-75.

Alves, F. G., de Sousa Carvalho, I. I. A., \& de Almeida, S. A. (2021). Síndrome do respirador oral e suas alterações dento faciais: uma revisão integrativa. Facit Business and Technology Journal, 1(26)137-149. 
Andrade, M. A., Moura, A. B. R., Medeiros, F. L. S. de, Matos, N. O., Goes, V. N., Gomes, L. L., Lima, F. de O., Cavalcanti, R. B. de M. S., Araújo Neto, A. P. de P. de, Alves, M. R. F., Alves, M. A. S. G., Penha, E. S. da, Guênes, G. M. T., \& Oliveira Filho, A. A. de. (2020). Relationship between oclusions and parafunctional habits in early childhood. Research, Society and Development, 9(7), e484974260. https://doi.org/10.33448/rsd-v9i7.4260

Angell, D. H. (1860). Treatment of irregularity of the permanent or adult teeth. Dental Cosmos, 1, 540-544.

Basualdo, A., Kramer, P. F., Fregapani, P. W., Perim, P. F., \& Fontanella, V. R. C. (2014). Avaliação longitudinal das alterações transversais após expansão rápida cirurgicamente assistida da maxila. Orthodontic Science and Practice, 7(26),160-165.

Bell, R. A. (1982). A review of maxillary expansion in relation to rate of expansion and patient's age. American Journal of Orthodontics, 81(1), 32-37.

Belluzzo, R. H. L., Faltin Junior, K., Lascala, C. E., \& Vianna, L. B. R. (2012). Maxillary constriction: are there differences between anterior and posterior regions?. Dental Press Journal of Orthodontics, 17, 1-6.

Bervian, J., Neiss, G., \& Zaffari, S. C. (2017). O conhecimento dos graduandos em odontologia acerca da atuação fonoaudiológica na motricidade orofacial. Revista Interdisciplinar, 10(1), 147-155.

Bruder, C., Ortolani, C. L. F., Lima, T. A. D., Artese, F., \& Faltin, K. (2019). Evaluation of palate area before and after rapid maxillary expansion, using conebeam computed tomography. Dental Press Journal of Orthodontics, 24, 40-45.

Caldas, L. D., Bittencourt, M. A. V., \& Torres, S. R. (2019). Complicações decorrentes da expansão rápida da maxila com aparelho dentomucossuportado: relato de quatro casos clínicos. Revista Clínica de Ortodontia Dental Press, 18(3)101-117.

Domann, J., Cruz, C. M., Crepaldi, M. V., Crepaldi, M. D. L. S., \& de Oliveira, B. L. D. S. (2017). Mordida aberta anterior, etiologia, diagnóstico e tratamento precoce. Revista Faipe, 6(2), 28-42.

Ferreira, G. da S.., Santos, LC dos., Meira, J. de F.., Martinho, RL de M.., Oliveira, NC da S. de., Santos, BRM dos., Meira, G . de F.., \& Rêgo, JTM. (2021). Tratamento ortopédico em paciente com deficiência maxilar: relato de caso. Research, Society and Development, 10(17), e127101724607. https://doi.org/10.33448/rsd-v10i17.24607

İş11, A. R. A. S., Ölmez, S., Akay, M. C., Günbay, T., \& Aynur, A. R. A. S. (2017). The effects of maxillary expansion on the soft tissue facial profile. Journal of Istanbul University Faculty of Dentistry, 51(3), 1-10.

Haas, A. J. (1961). Rapid expansion of the maxillary dental arch and nasal cavity by opening the midpalatal suture. The Angle Orthodontist, 31(2), 73-90.

Lima Filho, R. (2009). Alterações na dimensão transversal pela expansão rápida da maxila. Revista Dental Press de Ortodontia e Ortopedia Facial, 14, 146157.

Oliveira, N. L., Da Silveira, A. C., Kusnoto, B., \& Viana, G. (2004). Three-dimensional assessment of morphologic changes of the maxilla: a comparison of 2 kinds of palatal expanders. American Journal of Orthodontics and Dentofacial Orthopedics, 126(3), 354-362.

Pavithra, S., Rama, M., Revathi, E., \& Aruna, J. (2019). Rapid Maxillary Expansion and Appliance. Journal of Academy of Dental Education, 3(1), 1-4.

Pereira, A. S., Shitsuka, D. M., Parreira, F. J., \& Shitsuka, R. (2018). Metodologia da pesquisa científica. (pp. 3-9). UFSM. https://repositorio. ufsm. br/bitstream/handle/1/15824/Lic_Computacao_Metodologia-Pesquisa-Cientifica. pdf.

Neves, M. J. D., \& Itaborahy, W. M. (2008). Associação entre a Ortopedia Funcional e Ortodontia para o tratamento de mordida cruzada com assimetria facial: relato de caso clínico. Revista Clínica de Ortodontia Dental Press, 7(1)98-104.

dos Santos, A. M., Esper, A. B. M., Rocha, A. D., Lima, C. D. C. C., Batista, M. E., \& Holz, I. S. (2019). Expansão rápida da maxila assistida com miniimplantes (MARPE) em adultos. Parte I-aspectos gerais. Revista Clínica de Ortodontia Dental Press, 18(5)110-117.

Scariot, R., Junior, W. D. S. F., Morosini, I. C., dos Santos, K. M., Petinatti, M. F. P., Sebastiani, A. M., Rebellato, N. L. B., da Costa, D. J. (2020). Análise das modificações dentoesqueletais em pacientes submetidos à expansão rápida de maxila assistida cirurgicamente. Revista Sul-Brasileira de Odontologia RSBO, 17(1), 31-09.

Rabêlo, L. R. S., Bastos, E. G., Germano, A. R., \& Passeri, L. A. (2002). Expansäo de maxila cirurgicamente assistida sob anestesia local. Revista Dental Press de Ortodontia e Ortopedia Facial, 6(4),73-79.

Rech, R. S., Brown, M. A., Cardoso, M. C. D. A. F., Vidor, D. C. G. M., \& Maahs, M. A. P. (2015). Interfaces entre fonoaudiologia e odontologia: em que situações essas ciências se encontram? Universitas: Ciências da Saúde, 13(2), 111-125.

Ruiz, V. F., da Cruz, C. M., Ferreira, D., de Aguiar, A. P., \& da Silva, L. M. (2018). Expansão Rápida da Maxila. Revista Faipe, 7(2), 105-109. 